\section{Kutanes Leiomyosarkom}

Zusammenfassung. Bei einem 48-jährigen Patienten konnte histologisch ein kutanes Leiomyosarkom diagnostiziert werden, das ventral am Thorax aufgetreten war. Anamnestisch bestand der Tumor zum Zeitpunkt der Diagnose bereits seit etwa 7 Jahren. Die Staging-Untersuchungen ergaben keinen Hinweis für eine Metastasierung. Die Prognose der oberflächlichen Leiomyosarkome ist unter anderem abhängig von ihrer Lokalisation innerhalb der Hautstrukturen und bei den kutanen Formen günstiger als bei den subkutanen oder den viszeralen Manifestationsformen.

Cutaneous Leiomyosarcoma. A 48-year-old male patient presented with a sternally located skin tumor that histologically turned out to be a cutaneous leiomyosarcoma. The tumor had first been noted by the patient about 7 years ago. Further investigations showed no evidence of metastasis. The prognosis of superficial leiomyosarcomas depends on the localization within the skin (cutaneous vs. subcutaneous). The purely cutaneous tumors have a more favorable outlook than the subcutaneous or extracutaneous forms.

\section{Einleitung}

Die Leiomyosarkome sind maligne mesenchymale Neoplasien der glatten Muskulatur. Gemäß der Existenz glatter Muskelzellen in den jeweiligen anatomischen Strukturen finden sich Leiomyosarkome unter anderem retroperitoneal, seltener gastrointestinal, urogenital oder im Respirationstrakt. Auch die großen Gefäßsysteme, wie z.B. die V. cava oder die A. iliaca, sind bevorzugt betroffene Lokalisationen [19]. Die Prognose der viszeral auftretenden Leiomyosarkome ist im Allgemeinen ungünstig bis infaust. Häufig muss bereits bei der Diagnose des Primärtumors eine Metastasierung beobachtet werden $[18,19]$. Im Vergleich zu den Formen der viszeralen Manifestation zeichnen sich die primären Leiomyosarkome der Haut durch ein biologisch weniger aggressives Verhalten aus [3,12]. Dabei ist ihr Vorkommen insgesamt als selten anzusehen. Innerhalb der Gruppe der malignen Weichteiltumoren wird ihre

Akt Dermatol 2001; 27: 30-33

(c) Georg Thieme Verlag Stuttgart · New York ISSN 0340-2541

\author{
G. Wagner ${ }^{1}$, A. Barghorn ${ }^{2}$ \\ ${ }^{1}$ Hautklinik Zentralkrankenhaus Bremerhaven \\ (Chefarzt: Dr. G. Wagner) \\ ${ }^{2}$ Dermatologische Klinik Universitätsspital Zürich \\ (Direktor: Prof. Dr. G. Burg)
}

Häufigkeit mit 2,3-5,3\% angegeben [4,16]. Auf der Grundlage klinischer, histopathologischer und prognostischer Merkmale werden bei den Leiomyosarkomen der Haut kutane und subkutane Formen voneinander abgegrenzt. Innerhalb dieser Gruppe ist die Prognose der kutanen Leiomyosarkome wiederum günstiger als die der subkutanen Form [4].

Bei dem hier vorgestellten Patienten bestand das kutane Leiomyosarkom anamnestisch seit ca. 7 Jahren. Zum Zeitpunkt der Diagnose konnte keine Metastasierung festgestellt werden. Dies unterstreicht die relativ günstige Prognose der kutanen Leiomyosarkome.

\section{Kasuistik \\ Pat.: F. K., 48 Jahre, Tischler}

\section{Anamnese}

Der Patient berichtete über ein kleines Knötchen, das er erstmals vor etwa 7 Jahren an der rechten Brust bemerkt hatte. Hieraus entwickelten sich im Laufe der folgenden Jahre langsam, aber kontinuierlich an Größe zunehmende Knoten. Spontane oder druckbedingte Schmerzen im Bereich des Tumors verneinte der Patient. Die Vorstellung in der Hautklinik Bremerhaven erfolgte unter der Verdachtsdiagnose eines Dermatofibrosarcoma protuberans.

\section{Aufnahmebefund}

Pektoral rechts ein ca. $6 \times 3 \mathrm{~cm}$ großer, in der Tiefe gut abgrenzbarer und verschieblicher, an der Oberfläche aus linsenbis halbkirschgroßen Knoten aufgebauter Tumor von überwiegend hell- bis dunkelroter Färbung (Abb.1). Bei der Palpation fast steinharte Konsistenz. Übriger dermatologischer Befund unauffällig, keine Lymphknoten tastbar.

\section{Befunde diagnostischer Untersuchungen}

Histopathologischer Befund des Tumors

Mesenchymaler Tumor mit einer zentral liegenden, landkartenartig begrenzten Koagulationsnekrose. Spindelige bis ovaläre Tumorzellen mit eosinophilem Zytoplasma und länglichen bis spindeligen Zellkernen. Verschobene Kernplasmarelation, Anisokaryose. Deutliche mitotische Aktivität der Tu- 


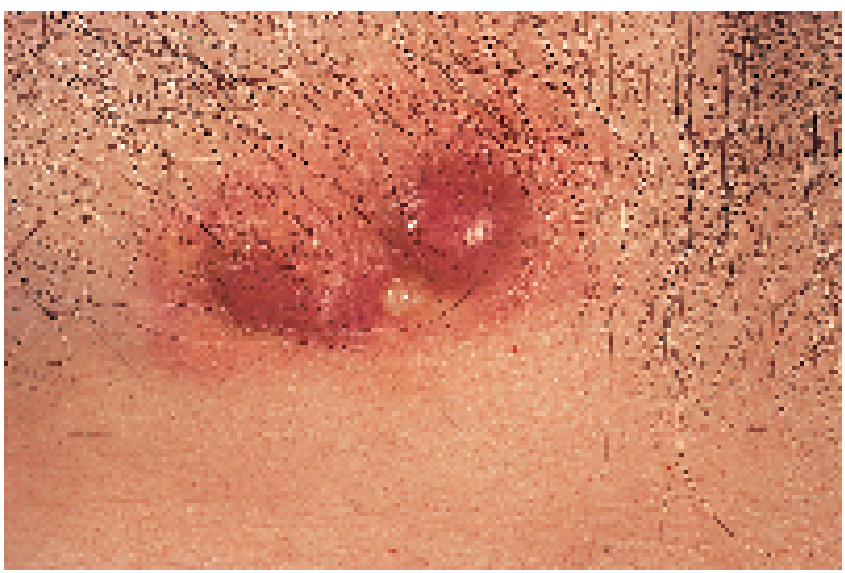

Abb. 1 Kutanes Leiomyosarkom pektoral rechts.

morzellen (9 Mitosen in 10 HPF). Tumorzellen in langgestreckten Faszikeln angeordnet, die sich orthogonal durchflechten. Punctum maximum der Tumorausdehnung im Bereich der Dermis, die bis in das Stratum papillare infiltriert ist. Der Tumor zeigt sich breitflächig in der gesamten Dermis und infiltriert die Subkutis vornehmlich entlang bindegewebiger Septen (Abb. 2 u. 3).

Immunhistochemie

Expression der Tumorzellen von glattmuskulärem Aktin (SMA) und des muskulären Intermediärfilaments Desmin.

Histopathologischer Befund des Lymphknotens Achsel rechts

Unspezifisch entzündlicher, durchweg tumorfreier Lymphknoten.

\section{Laborbefunde}

Die routinemäßig bestimmten Laborparameter waren ohne pathologischen Befund.

\section{Ergänzende Untersuchungen}

Rö.-Thorax, Sonographie Abdomen und Achseln bds. ohne pathologischen Befund. Ebenfalls unauffällige Ganzkörperszintigraphie. In der Computertomographie zeigt sich axillär rechts ein $2 \mathrm{~cm}$ durchmessender, suspekt wirkender Lymphknoten. Die übrigen Befunde der CT-Untersuchung waren unauffällig.

\section{Therapie und Verlauf}

Der Tumor wurde primär in toto weit im Gesunden exzidiert. Nach Vorlage des CT-Befundes mit Nachweis eines suspekten Lymphknotens in der rechten Achsel erfolgte hier eine selektive Lymphadenektomie, ohne dass eine Metastasierung histopathologisch nachgewiesen werden konnte. Der postoperative Verlauf war unauffällig.

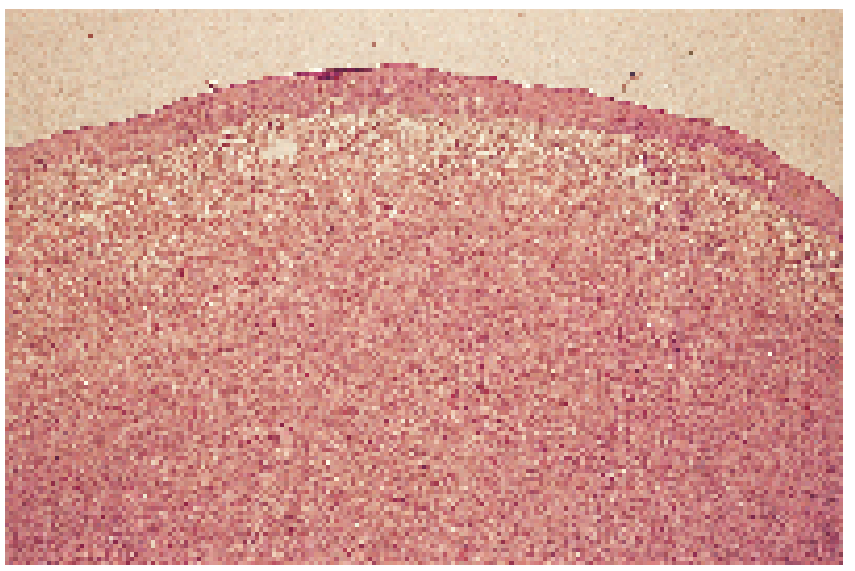

Abb. 2 Histologische Übersicht: Spindelige Neoplasie der Dermis mit faszikulärem Wachstumsmuster (HE × 25).

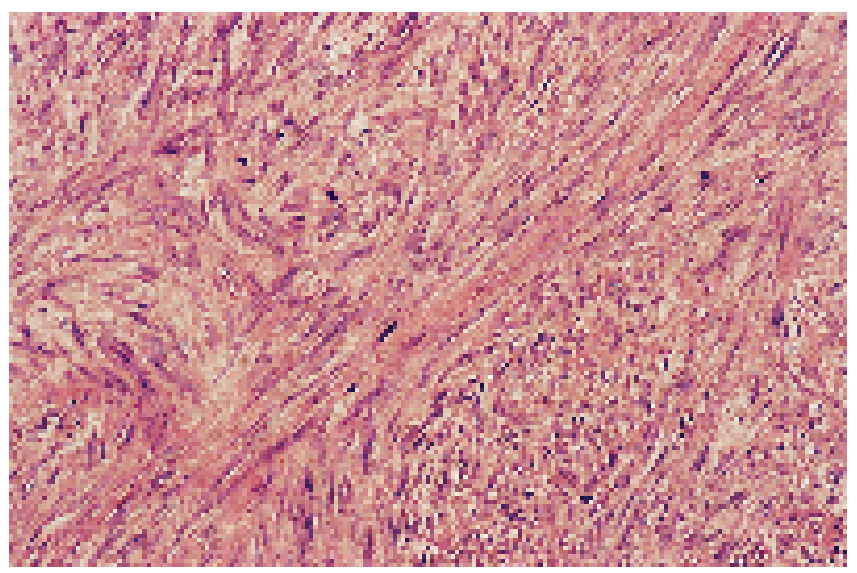

Abb. 3 Detail aus Abb. 2: Spindelige Zellformationen in Bündeln angeordnet, sich durchflechtend, mit stumpf endenden, zigarrenförmigen Zellkernen. Auffällig ist die hohe Mitoserate (HE × 200).

\section{Diskussion}

Die Leiomyosarkome der Haut, die auch als oberflächliche Leiomyosarkome bezeichnet werden, sind selten. Innerhalb der Gruppe maligner Weichteilneoplasien liegt der Anteil ihrer Häufigkeit zwischen 2,3 und 5,3\% [4,16]. Bei der Beurteilung ihrer epidemiologischen Merkmale muss das seltene Vorkommen dieser Tumorformen berücksichtigt werden, wodurch sich teilweise abweichende oder auch widersprüchliche Angaben zu einzelnen Aspekten der Epidemiologie erklären lassen. So finden sich in der Literatur überwiegend kasuistische Beiträge zum Thema. Nur vereinzelt konnten die Befunde größerer Patientenkollektive retrospektiv ausgewertet werden $[2,4,13]$. Bei der Altersverteilung herrscht Einigkeit darüber, dass die oberflächlichen Leiomyosarkome in jedem Alter auftreten können, vereinzelt sogar auch bei Kindern oder Neugeborenen [7,10]. Die Frage, in welchem Lebensabschnitt die Leiomyosarkome der Haut am häufigsten entstehen, wird hingegen unterschiedlich beurteilt. Während einzelne Autoren den Tumor als ein Malignom der älteren Menschen mit einem Häufigkeitsgipfel im 6. und 7. Dezennium ansehen, zeigen andere Untersuchungen eine Bevorzugung des mittleren Lebensalters oder auch eine gleichmäßige Häufigkeitsverteilung zwischen dem 30. und 70. Lebensjahr [7,8,15,18]. Die Angaben zur 
Geschlechtsverteilung variieren ebenfalls deutlich. Neben einem ausgeglichenen Verhältnis der Manifestation zwischen beiden Geschlechtern finden sich in der Literatur auch Untersuchungen, bei denen Frauen oder Männer bevorzugt betroffen gewesen sind $[7,8,14,16]$.

Die klinische Morphologie der oberflächlichen Leiomyosarkome ist abhängig von ihrer Entstehung und Lokalisation innerhalb der Hautstrukturen. Dabei unterscheidet man kutane und subkutane Formen des Tumors, wobei die kutane Manifestation deutlich häufiger beobachtet wird als die subkutane [7]. Die kutanen Leiomyosarkome, die histogenetisch überwiegend von den $\mathrm{Mm}$. arrectores pilorum abstammen, zeigen im Allgemeinen ein nur sehr langsames Wachstum. Als Konsequenz dieser Wachstumsdynamik weist die Mehrzahl der kutanen Leiomyosarkome zum Zeitpunkt ihrer Diagnose einen relativ kleinen Durchmesser auf, der trotz häufig jahrelanger Anamnese im allgemeinen nur $2-3 \mathrm{~cm}$ beträgt [7]. In der Regel besteht der Tumor aus einem oder mehreren miteinander verwachsenen Knoten. Dieser knotige, das Hautniveau überragende Anteil kann mit einer in der Tiefe lokalisierten, größeren plattenartigen Basis verbunden sein. Die Oberfläche des kutanen Leiomyosarkoms zeigt typischerweise eine rötliche, rötlich-livide oder bräunliche Verfärbung. Die Epidermis ist mit dem Tumor verbacken und somit nicht frei verschieblich. Hämorrhagische Krusten oder blutende Ulzerationen zählen zu den weiteren klinischen Merkmalen der kutanen Leiomyosarkome. Die subkutane Variante des Leiomyosarkoms, deren Ursprung in der glatten Muskulatur subkutaner Gefäße gesehen wird, ist zum Zeitpunkt der Diagnose im Allgemeinen größer als die kutane Form. Nicht selten werden Tumordurchmesser von über $7 \mathrm{~cm}$ erreicht [7]. Auch die subkutanen Leiomyosarkome stellen sich klinisch als knotige, meist gut abgrenzbare Strukturen dar, die jedoch in der Tiefe lokalisiert sind und häufig eine hautfarbene Oberfläche zeigen mit hier frei verschieblicher Epidermis. Regressive Oberflächenveränderungen gehören nicht zum typischen klinischen Befund $[3,15]$. Zu den klinischen Gemeinsamkeiten der beiden Varianten oberflächlicher Leiomyosarkome zählen ihr solitäres Auftreten, eine derbe bis knochenharte Konsistenz und spontan auftretende oder druckprovozierbare Schmerzen im Tumorbereich [7,18]. Auch bei der Lokalisation am Integument finden sich keine Unterschiede zwischen den beiden Formen. Am häufigsten betroffen sind die Streckseiten der unteren Extremitäten, gefolgt von den oberen Extremitäten und dem Stamm [7,8].

Histopathologisch zeichnen sich die Leiomyosarkome der Haut durch langgestreckte, spindelförmige oder polymorphe Zellen aus, die in Bündeln angeordnet ein dreidimensional verflochtenes Netzwerk bilden. Die Zellkerne zeigen ein unregelmäßig verteiltes Chromatin und sind an ihren Enden typischerweise zigarrenförmig abgerundet. Größe, mehrkernige Riesenzellen und unregelmäßig verteilte Mitosen stellen weitere Kriterien der Malignität dar [6,11]. Immunhistochemisch exprimieren die Tumorzellen typischerweise das glattmuskuläre Aktin sowie die Intermediärfilamente Vimentin und Desmin, wobei im Einzelfall Abweichungen der Befundkonstellationen möglich sind [17]. So fanden Kaddu et al. bei 19 oberflächlichen Leiomyosarkomen in allen Fällen die Expression von glattmuskulärem Aktin und Vimentin, während nur in 15 Fällen das panmuskuläre Aktin (HHF-35) und nur bei 12 Tumoren Desmin nachweisbar waren. Darüber hinaus wiesen einige Leiomyosarkome zusätzlich die Expression von Zytokeratinen und S100 auf, was bezüglich der histologischen Differenzialdiagnosen von Bedeutung sein kann [13]. Die Prognose der oberflächlichen Leiomyosarkome ist deutlich günstiger als die der viszeralen Formen $[18,19]$. Darüber hinaus bestehen aber auch innerhalb der Gruppe oberflächlicher Leiomyosarkome prognostisch Unterschiede: Neben der Tumorgröße und der Lokalisation innerhalb der Hautstrukturen - hierbei sind die Manifestation und eine große Ausdehnung des Tumors in der Subkutis als ungünstige Kriterien zu werten - wird histopathologisch als verlässlichstes Malignitätskriterium die Mitoserate pro $10 \mathrm{HPF}$ angesehen, wobei der cut-off-Punkt mit $1 \mathrm{MF} /$ $10 \mathrm{HPF}$ oder mit $5 \mathrm{MF} / 10 \mathrm{HPF}$ angegeben wird [6,7]. Während die kutanen Leiomyosarkome eine Rezidivrate von etwa $40 \%$ aufweisen, jedoch nur in Einzelfällen metastasieren, zeigen die subkutanen Leiomyosarkome in ca. 50\% Lokalrezidive und in bis zu 33\% Metastasen [7,17]. Bei der im Allgemeinen hämatogenen Metastasierung sind neben Lunge, Leber und Gehirn auch kardiale und osteogene Metastasen beobachtet worden. Nur sehr selten wurde eine lymphogene Ausbreitung des Tumors beschrieben $[12,16,17]$. Die Therapie der Wahl oberflächlicher Leiomyosarkome ist die Exzision weit im Gesunden $[3,5,15]$. Auch vor dem Hintergrund der lokalen Rezidivraten ist eine engmaschige postoperative Überwachung der Patienten notwendig. Neben der operativen Therapie sind andere Behandlungsverfahren nur selten beschrieben worden $[1,12]$. Allerdings wurde erst kürzlich über die komplette Remission eines kutanen Leiomyosarkoms der Knöchelregion berichtet, die durch isolierte Extremitätenperfusion mit TNF-alpha und Melphalan erreicht werden konnte [9].

\section{Literatur}

${ }^{1}$ Armijo M, Herrera E, De Dulanto F, Naranjo R, Camacho F. Leiomyosarcome de la verge. Étude ultrastructurale. Ann Dermatol Venereol (Paris) 1978; 105: 267 - 274

${ }^{2}$ Auroy S, Contesso G, Spatz G, Genin J, Margulis A, Lecesne A, Avril MF. Primary cutaneous leiomyosarkoma: 32 cases. Ann Dermatol Venereol 1999; 126: 235-242

3 Braun-Falco O, Kind P, Meister P. Kutanes Leiomyosarkom. Hautarzt 1984; 35: $428-429$

${ }^{4}$ Dahl I, Angervall L. Cutaneous and subcutaneous Leiomyosarcoma: a clinicopathologic study of 47 patients. Pathol Eur 1974; 9: 307-315

${ }^{5}$ Davidson LL, Frost ML, Hanke W, Epinette WW. Primary leiomyosarcoma of the skin. Case report and review of the literature. J Am Acad Dermatol 1989; 21: 1156-1160

${ }^{6}$ Enzinger FM, Weiss SW. Soft tissue tumors. 3. Aufl. St. Louis: Mosby, 1995

${ }^{7}$ Fields JP, Helwig EB. Leiomyosarcoma of the skin and subcutaneous tissue. Cancer 1981; 47: 156-169

${ }^{8}$ Hashimoto H, Daimaru Y, Tsuneyoshi M, Enjoji M. Leiomyosarcoma of the external soft tissues. A clinicopathologic, immunhistochemical and electron microscopic study. Cancer 1986; 57: 2077-2088

${ }^{9}$ Häffner AC, Zepter K, Fritz T, Dummer R, Lejeune FJ, Burg G. Complete remission of advanced cutaneous leiomyosarcoma following isolated limb perfusion with high-dose tumour necrosis factor alpha und melphalan. Br J Dermatol 1999; 141: 935 - 936

${ }^{10}$ Heieck JJ, Organ CH. Leiomyosarcoma of the scalp in a newborn. Arch Dermatol 1970; 102: 213-215

${ }^{11}$ Hornstein OP, Weidner F. Tumoren der Haut. In: Doerr W, Seifert G, Uehlinger E (Hrsg). Spezielle pathologische Anatomie, Band 7, Teil 2. Berlin, Heidelberg, New York: Springer, 1979

${ }^{12}$ Jegasothy BV, Gilgor RS, Hull M. Leiomyosarcoma of the skin and subcutaneous tissue. Arch Dermatol 1981; 117: 478-481 
${ }^{13}$ Kaddu S, Beham A, Cerroni L, Humer-Fuchs U, Salmhofer W, Kerl H, Soyer HP. Cutaneous leiomyosarcoma. Am J Surg Pathol 1997; 21: $979-987$

${ }^{14}$ Phelan JT, Scherer M, Mesa P. Malignant smooth-muscle tumors (leiomyosarcomas) of soft-tissue origin. N Engl J Med 1962; 266: $1027-1030$

${ }^{15}$ Stieler W, Mensing H, Schaeg G, Jehn E. Primäres kutanes Leiomyosarkom. Hautarzt 1991; 42: 44-47

${ }^{16}$ Stout AP, Hill WT. Leiomyosarcoma of the superficial soft tissues. Cancer 1958; 11: 844-854

17 Swanson PE, Stanley MW, Scheithauer BW, Wick MR. Primary cutaneous leiomyosarcoma. A histological and immunhistochemical study of 9 cases, with ultrastructural correlation. J Cutan Pathol 1988; 15: 129-141

${ }^{18}$ Wascher RA, Lee MYT. Recurrent cutaneous leiomyosarcoma. Cancer 1992; 70: 490-492

${ }^{19}$ Wile AG, Evans HL, Ronsdahl MM. Leiomyosarcoma of soft tissue: A clinicopathologic study. Cancer 1981; 48: 1022-1032

\section{Dr. G. Wagner}

Hautklinik Zentralkrankenhaus Reinkenheide

Postbrookstraße 103

27574 Bremerhaven 